\title{
What we can see from very small size sample of metagenomic sequences
}

\author{
Jaesik Kwak ${ }^{\top}$ and Joonhong Park ${ }^{2^{*}}$
}

\begin{abstract}
Background: Since the analysis of a large number of metagenomic sequences costs heavy computing resources and takes long time, we examined a selected small part of metagenomic sequences as "sample"s of the entire full sequences, both for a mock community and for 10 different existing metagenomics case studies. A mock community with 10 bacterial strains was prepared, and their mixed genome were sequenced by Hiseq. The hits of BLAST search for reference genome of each strain were counted. Each of 176 different small parts selected from these sequences were also searched by BLAST and their hits were also counted, in order to compare them to the original search results from the full sequences. We also prepared small parts of sequences which were selected from 10 publicly downloadable research data of MG-RAST service, and analyzed these samples with MG-RAST.

Results: Both the BLAST search tests of the mock community and the results from the publicly downloadable researches of MG-RAST show that sampling an extremely small part from sequence data is useful to estimate brief taxonomic information of the original metagenomic sequences. For 9 cases out of 10, the most annotated classes from the MG-RAST analyses of the selected partial sample sequences are the same as the ones from the originals.

Conclusions: When a researcher wants to estimate brief information of a metagenome's taxonomic distribution with less computing resources and within shorter time, the researcher can analyze a selected small part of metagenomic sequences. With this approach, we can also build a strategy to monitor metagenome samples of wider geographic area, more frequently.
\end{abstract}

Keywords: Metagenomics, Sampling, Mock community, MG-RAST, BLAST

\section{Background}

As next-generation sequencing is getting popular [1], a large number of genome sequences now can be easily generated for metagenomics research [2]. However, since analyzing a large number of sequences usually costs heavy computing resources and takes long time [3].

To shorten computation time and reduce requirements for computing resources, researchers introduced advanced algorithmic techniques and database optimization methods. MetaPhlAn uses a database engineered to contain specific marker genes to do sequence classification quickly [4]. Kraken searches a large k-mer database designed for its own search method to look up its taxonomic trees [5]. Centrifuge focuses more on compression of database sequences to reduce the size of database to search [6].

\footnotetext{
* Correspondence: parkj@yonsei.ac.kr

${ }^{2}$ School of Civil and Environmental Engineering, Yonsei University, 50 Yonsei

Ro, Seodaemun Gu, Seoul 038722, South Korea

Full list of author information is available at the end of the article
}

On the other hand, there have been several different ways to get information only from a relatively small part of the available data [7].

One example to reduce the cost of sequencing and computing was a study to get an optimal depth of sequencing for16s rRNA [8]. This study demonstrated that a small number of Illumina single-end reads, such as 2000 reads, were enough to recapture the taxonomy information and diversity patterns. It showed a possibility that meaningful information can be derived even from a small portion of full sequences. However, it was tested only for a certain type of gene, 16S rRNA [9].

Another example was "genome skimming" study that showed the simulation results of rDNA assembly from shallow sequencing of plant genomes [10]. Based on the efficiency of the shallow sequencing that identified the low-copy fraction of the nuclear genome, this study suggested a strategy, where there are multiple candidate species of interest, using shallow sequencing to choose a 
species with the best condition, before using deeper sequencing of that chosen species to know more details.

This concept of genome skimming is also applicable to metagenome. One study pointed out that "metagenome skimming" can be an efficient tool to capture "the genomic diversity of poorly studied, species-rich lineages", after analyzing the sequencing results on two pools of Coleopteran, that consisted about 200 species [11]. However, both studies targeted eucaryocyte and used assembly method to analyze taxonomy, that still requires long computation time for assembling process and a large amount of sequences, which were more than hundred thousands of reads.

\section{Aims and objectives}

In this study, getting taxonomic information from small size sample of a large metagenome sequence data was examined, in order to save computing resources and to shorten processing time.

We utilized a simple rarefaction technique, often used for various studies such as determination of optimal sequencing depth [12]. We applied it to estimating brief taxonomic information from extremely small parts of various metagenomic sequences. We wanted to find out how realistic that the extraction of taxonomic information from those small parts is in practical cases. If it is a practical approach, we might develop a protocol or a standard to preview or pre-check metagenomic sequences with a quick estimation before doing a full-scale analysis for them.

We selected a small part of metagenomic sequences in several ways. We treated these selected sequences as a sample of original full sequences. The phylum and the class with dominant populations were annotated in the sample and compared to ones annotated in the original full sequences, since they are generally considered as important information in metagenomics [13]. The diversities of phyla and classes were also compared.

A mock community, which was intentionally made of known bacterial strains to get a mixed genome, was examined with BLAST search [14]. Since we know the real taxonomic information of the mock community, we can evaluate how well the samples that we made represent the original taxonomic information. We also applied this approach to known results of existing researches, which are available publicly in MG-RAST web site, which has been an open access web service widely used for metagenomics analysis [15].

\section{Results}

\section{Mock community}

The original full sequences obtained from the mock community of 10 strains were about $1,220,000$ reads or
12.3 Gb. The GC content calculated from them was $53.1 \%$.

The results of the GC content calculation for 176 different samples, which were selected from the original full sequences by 16 different selection types for each of 11 different sample sizes from 100 to 50,000 reads, show that GC content values get closer to $53.1 \%$, as the sizes of the samples increase. (Fig. 1) This can be regarded as supportive evidence that a sample with a large enough size represents the nature of the original full sequences.

To analyze the taxonomy of this mock community, the numbers of hit reads from BLAST search for each of 10 strains of the original full sequences were counted. Their ratios to the sum of all 10 strains' hits range from 0.014 to 0.186 . (Table 1) These are the original values that we want to estimate with BLAST searches.

In order to do the estimation, the ratio of hit reads counted for each strain to the sum of all 10 strains' hits was calculated for each of the 176 different samples, again, which were selected from the original full sequences by 16 different selection types per 11 different sample sizes.

The result of the calculation from the samples shows that the ratio values for Roseobacter get closer to 0.057, which was the ratio value of Roseobacter calculated from the original full sequences, as the sizes of the samples increase. The ratio values for Arthrobacter get closer to 0.014 similarly. (Fig. 2) The ratio values calculated for the samples of the other strains also show the similar results. To show the tendency that the deviation from the different sampling methods decreases while the size of the sample increases, the smallest values (Additional file 1: Table S1) and the largest values (Additional file 1: Table S2) among the ratio values calculated from 16 samples of each sample size were tabulated. The standard deviation values out of the ratios calculated from 16 samples of each sample size were also tabulated. (Additional file 1: Table S3).

As the size of the sample increases, the smallest values and the largest values show their tendency of getting closer to the ratio values calculated from the original full sequences. At the same time, as the size of the sample increases, the standard deviation value mainly decrease, though there are a few exceptions, since there are relatively large statistical errors where the values are small.

Again, these results support the tendency that a sample with a large enough size has its hit ratios that are close to ones of the original full sequence.

This means that small part of the original full sequences can be used to estimate original taxonomic annotation regardless of selection type, especially for relative comparison, such as to answer a question of which class is annotated most, and a question of which phylum is more annotated than another phylum. 


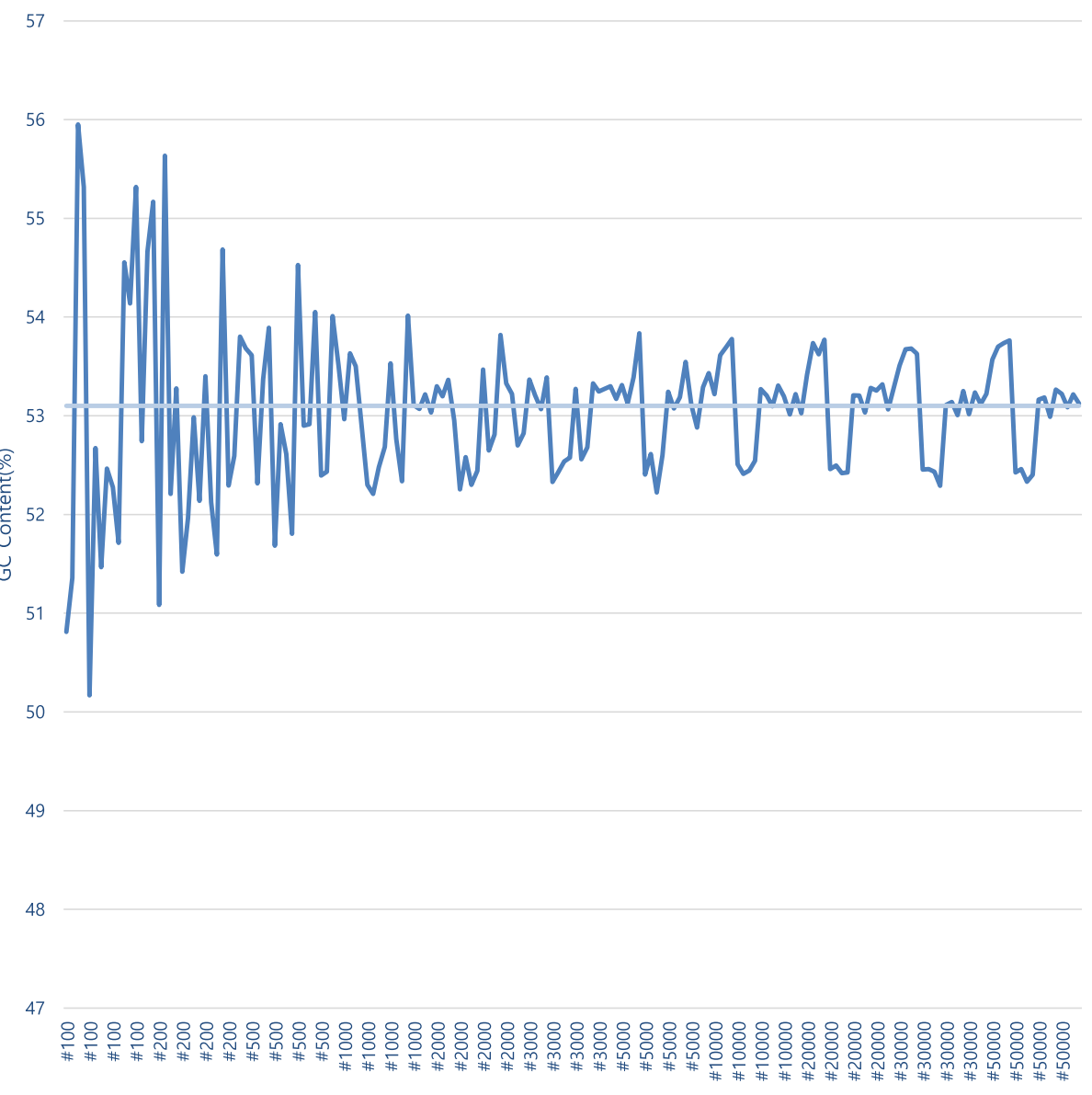

GC content -53.1

Fig. $1 \mathrm{GC}$ content of samples (The labels of $\mathrm{x}$-axis mean the sample sizes. They are placed per one selection type. This means a label represents 4 samples made by 4 different $K$ numbers)

Table 1 Hits of BLAST searches in the original full sequences of the mock community

\begin{tabular}{lll}
\hline Strain & Number of Hits & $\begin{array}{l}\text { Ratio: Number of hits for each } \\
\text { strain/Sum(=164,662,612) }\end{array}$ \\
\hline Escherichia coli KCTC 2571 & $30,658,032$ & 0.1862 \\
Escherichia coli Strain W & $29,390,176$ & 0.1785 \\
Staphylococcus epidermidis ATCC & $18,862,322$ & 0.1146 \\
Pseudomonas stutzeri ATCC 17588 & $18,559,245$ & 0.1127 \\
Klebsiella pneumoniae KCTC 2242 & $15,708,328$ & 0.0954 \\
Chromobacterium violaceum ATCC 12472 & $15,466,319$ & 0.0939 \\
Polaromonas naphthalenivorans CJ2 & $14,081,217$ & 0.0855 \\
Corynebacterium glutamicum ATCC 13032 & $10,351,377$ & 0.0629 \\
Roseobacter denitrificans OCh114 & $9,332,359$ & 0.0567 \\
Arthrobacter chlorophenolicus A6 & $2,253,237$ & 0.0137 \\
Sum & $164,662,612$ & 1 \\
\hline
\end{tabular}




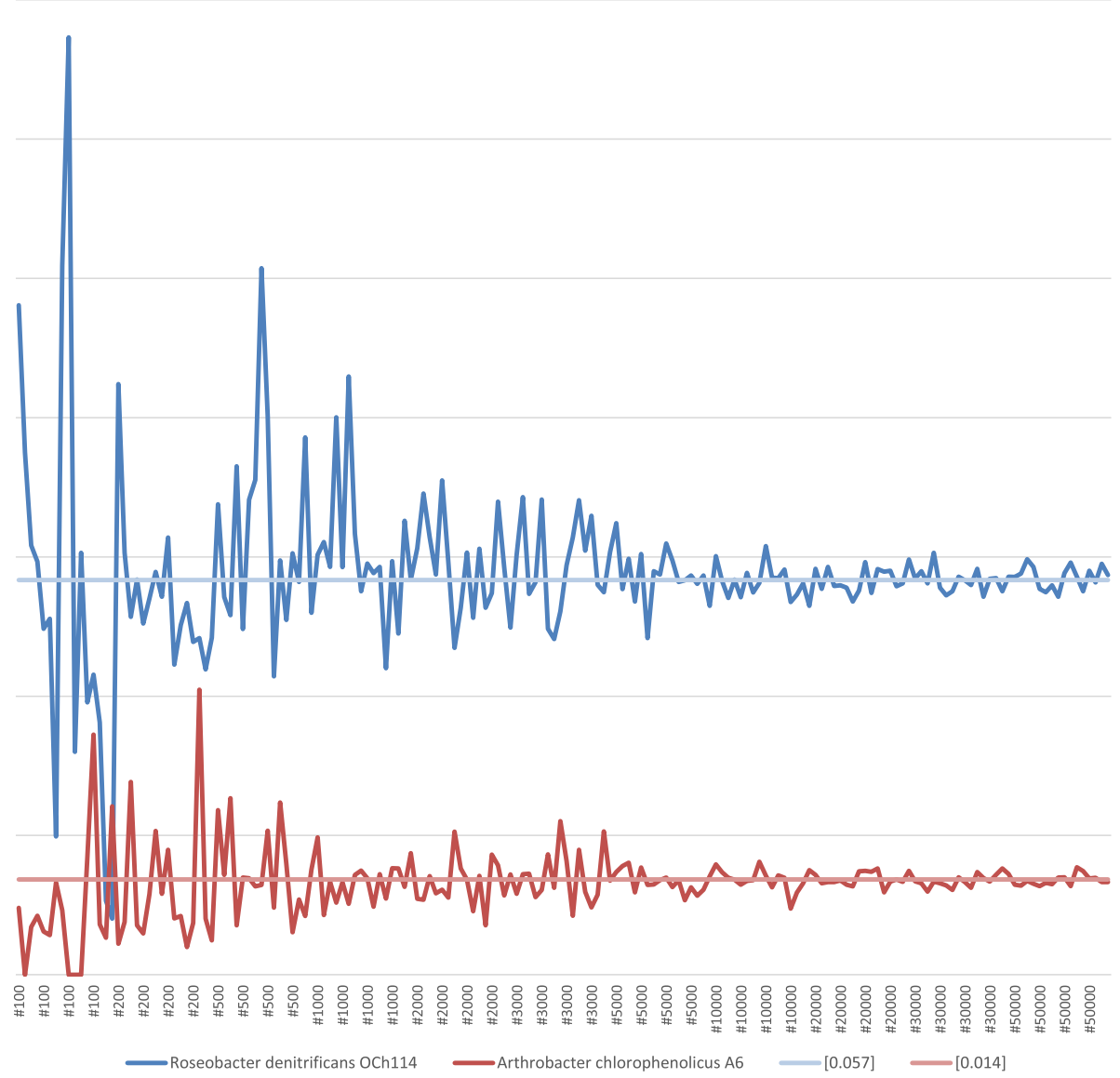

Fig. $2<$ Hit for strain/sums of all Hits> for each sample (The labels of x-axis mean the sample sizes. They are placed per one selection type. This means a label represents 4 samples made by 4 different $K$ numbers)

Meanwhile, we can explain the difference between the results from the original full sequences and the ones from the samples as a general statistical error problem of a small size sample.

For a given margin of error, we can approximate a proper sample size, if we consider that estimating a taxonomic proportion of sequences is similar to a general statistical sampling problem, such as a poll to estimate a proportion of voters to an election candidate.

For example, as a rough approximation, if we assume that a given unknown set of metagenomic sequences follows a normal distribution and expected proportion of reads classified as a certain taxon is close to $1 / 2$, which is a widely used value where we do not have any initial information about the actual proportion and the start-up cost of sampling is expensive [16], there is a simplified equation to calculate the size of the sample for a margin of error. (Eq. 1.) [16] By this calculation, the sample size for $1 \%$ margin of error and $85 \%$ confidence is about 5000 (5184).

$$
\mathrm{n}=\frac{(\mathrm{Z} \alpha / 2)^{2} \cdot \frac{1}{2} \cdot \frac{1}{2}}{\mathrm{E}^{2}}
$$

Eq. 1. Determining the sample size $\mathrm{n}$ in estimation of population proportion, where the probability of the range greater than $\mathrm{Z} \alpha / 2$ at the standard normal distribution equals to (1-confidence)/2, and $\mathrm{E}$ is margin of error.

If we apply this margin of error calculation to the mock community test, the result from this margin of error calculation might be smaller than the actual errors, because all the ratio values of the mock community from the original full sequences are smaller than $1 / 2$. Nevertheless, BLAST search result from a sample made by selecting 5000 reads from the start of the original full 
sequences ("selection type 1 " and 0 as "K number") of this mock community still gives fair estimation of the ratio values. (Fig. 3).

We can compare this to a more general case of statistical sampling problem. For instance, we made the sample whose size is 5000 reads to estimate total 1.22 million reads. On the other hand, New York Times/CBS News performed a poll of 1426 people for 2016 U.S. Presidential election of total 137 million voters [17].

\section{MG-RAST: Applicability in full-scale metagenomics sequencedata sets}

All the GC content values calculated from the original full sequences of the 10 public MG-RAST projects, that all have more than $170,000,000$ reads, were compared to the GC content values calculated from the samples, that only have 5000 reads selected from them. (Table 2) In most cases, the GC content values calculated from the samples estimate the ones calculated from the originals well.

The most annotated phyla and classes from the original MG-RAST research data were compared to the ones of the samples. (Table 3) For 9 cases out of 10, the most annotated phyla from the MG-RAST projects of the samples show the same results as the ones of the original data. For 9 cases out of 10, the most annotated classes are the same between the original MG-RAST research data and the ones of the samples. Considering that 4 different classes were shown among all the cases, these 9 out of 10 matches support the assumption that these samples can estimate the brief taxonomic information of the originals.

On the other hand, the numbers of the annotated phyla from the samples tend to be smaller than the ones from the originals. (Fig. 4) The numbers of the annotated classes from the samples tend to be even much smaller than the ones from the samples. (Fig. 5) These are because the samples did not include different sequences representing all the different phyla and classes in the original data. A phylum or a class that presents only a small number of sequences in original has low probability of being captured in a sample. This implies that this type of sampling cannot take all the taxonomic diversity information.

However, if we apply $1 \%$ threshold to remove over-annotation and/or mis-annotation, the numbers of the annotated phyla from the samples get much closer to the ones from the original data. (Fig. 6) The numbers of the annotated classes from the samples also get closer to the ones from the original data. (Fig. 7) This supports the assumption that this samples still can estimate, at least, part of taxonomic diversity information.

\section{Discussion}

Both the BLAST search tests of the mock community and the results from the publicly downloadable data sets of MG-RAST show that the sampling very small part of sequence data is useful to estimate the brief taxonomic information of the original metagenomic sequences. The sample sequences with their sizes of only 5000 reads,

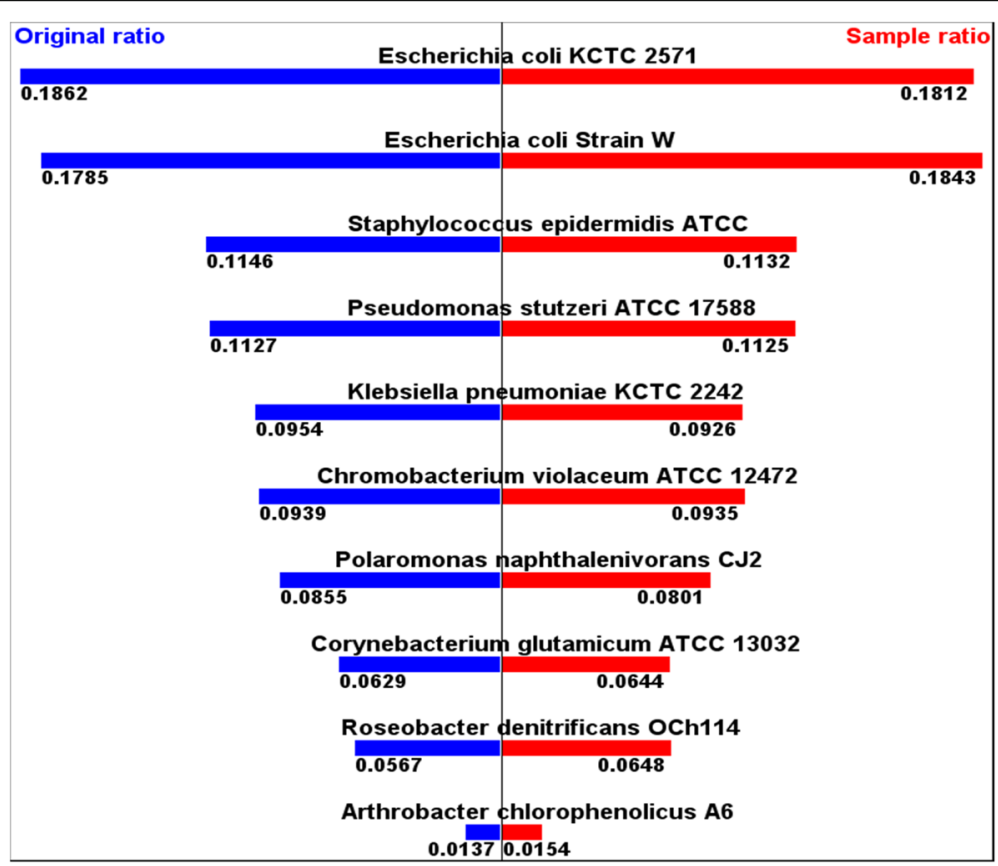

Fig. $\mathbf{3}$ < Hits of strain/sums of all hits $>$ from original and from sample with 5000 Reads 
Table 2 GC Contents, original vs. sample

\begin{tabular}{llll}
\hline Original MG-RAST ID & Sample MG-RAST ID & Original GC Content (\%) & Sample GC Content (\%) \\
\hline $4,539,528.3$ & $4,701,886.3$ & 62.975 & 62.161 \\
$4,510,219.3$ & $4,701,884.3$ & 53.813 & 51.365 \\
$4,510,173.3$ & $4,701,887.3$ & 50.512 & 50.477 \\
$4,509,400.3$ & $4,701,883.3$ & 62.269 & 62.468 \\
$4,562,385.3$ & $4,701,888.3$ & 56.816 & 57.733 \\
$4,538,997.3$ & $4,701,892.3$ & 58.078 & 56.902 \\
$4,539,575.3$ & $4,701,885.3$ & 60.177 & 60.568 \\
$4,587,432.3$ & $4,701,891.3$ & 52.943 & 52.68 \\
$4,555,915.3$ & $4,701,890.3$ & 48.254 & 48.132 \\
$4,533,611.3$ & $4,701,889.3$ & 56.184 & 45.012
\end{tabular}

selected from the large sequence data from the existing public cases of MG-RAST, give a useful estimation both to a question of what the most annotated phylum/class is and to a question of how diverse phyla/classes are.

On average, the size of the sample is only $0.002 \%$ of the original data, in terms of number of bases. This small size reduces computing time in MG-RAST from several months to a few hours.

It means we can get an estimated result of metagenomic sequence analysis quickly even with less computing resources when we use a small part of genome data. This aligns with the conclusions of shallow sequencing and the results of metagenome skimming to do an efficient analysis with less sequencing.

On the contrary, In the case where the sample estimates the most annotated phylum incorrectly (MG-RAST ID:4587432.3), the difference between the number of the most annotated phylum (Firmicutes) and the number of the second most annotated phylum (Actinobacteria) in the original is only $0.8 \%$ point. (Fig. 8 ) This small difference is the reason why the estimation from the sample is incorrect. Similarly, in the case where the sample estimates the most annotated class incorrectly (MG-RAST
ID:4538997.3), the difference between the number of the most annotated class (Alphaproteobacteria) and the number of the second most annotated class (Deltaproteobacteria) in the original is also as small as $2.2 \%$ point. (Fig. 9) These can be regarded as statistical errors. It means an analysis from a sample cannot identify a difference that is smaller than a certain statistical limit.

There is also possibility that the sampling method used here was not the optimal choice. Since our choice of the sampling method was just for minimizing the sampling cost, ignoring quality difference of different sampling methods. If we had tried any pre-checks for different sampling methods, such as comparing GC content values from different sampling methods with GC content of the original data, and tried to find a better sampling method among them, then it could have decreased the error.

On the other hand, the results of the most annotated phyla from MG-RAST tests are Proteobacteria for 8 out of 10 cases and the set of the most annotated classes has only 3 different classes. This is because the metagenomics research data we tested here were chosen only by their original sequence sizes, without any consideration

Table 3 Most annotated phylum and classes, original vs. sample

\begin{tabular}{llllll}
\hline Original MG-RAST ID & Sample MG-RAST ID & $\begin{array}{l}\text { Most Annotated } \\
\text { Phylum of Original }\end{array}$ & $\begin{array}{l}\text { Most Annotated } \\
\text { Phylum of Sample }\end{array}$ & $\begin{array}{l}\text { Most Annotated } \\
\text { Class of Original }\end{array}$ & $\begin{array}{l}\text { Most Annotated } \\
\text { Class of Sample }\end{array}$ \\
\hline $4,539,528.3$ & $4,701,886.3$ & Proteobacteria & Proteobacteria & Actinobacteria (class) & Actinobacteria (class) \\
$4,510,219.3$ & $4,701,884.3$ & Proteobacteria & Proteobacteria & Deltaproteobacteria & Deltaproteobacteria \\
$4,510,173.3$ & $4,701,887.3$ & Proteobacteria & Proteobacteria & Gammaproteobacteria & Gammaproteobacteria \\
$4,509,400.3$ & $4,701,883.3$ & Proteobacteria & Proteobacteria & Actinobacteria (class) & Actinobacteria (class) \\
$4,562,385.3$ & Proteobacteria & Proteobacteria & Gammaproteobacteria & Gammaproteobacteria \\
$4,538,997.3$ & $4,701,888.3$ & Proteobacteria & Proteobacteria & Alphaproteobacteria & Deltaproteobacteria \\
$4,539,575.3$ & $4,701,892.3$ & Proteobacteria & Proteobacteria & Alphaproteobacteria & Alphaproteobacteria \\
$4,587,432.3$ & $4,701,885.3$ & Firmicutes & Actinobacteria & Actinobacteria (class) & Actinobacteria (class) \\
$4,555,915.3$ & $4,701,891.3$ & Ascomycota & Ascomycota & Gammaproteobacteria & Gammaproteobacteria \\
$4,533,611.3$ & $4,701,890.3$ & Proteobacteria & Proteobacteria & Alphaproteobacteria & Alphaproteobacteria \\
\hline
\end{tabular}




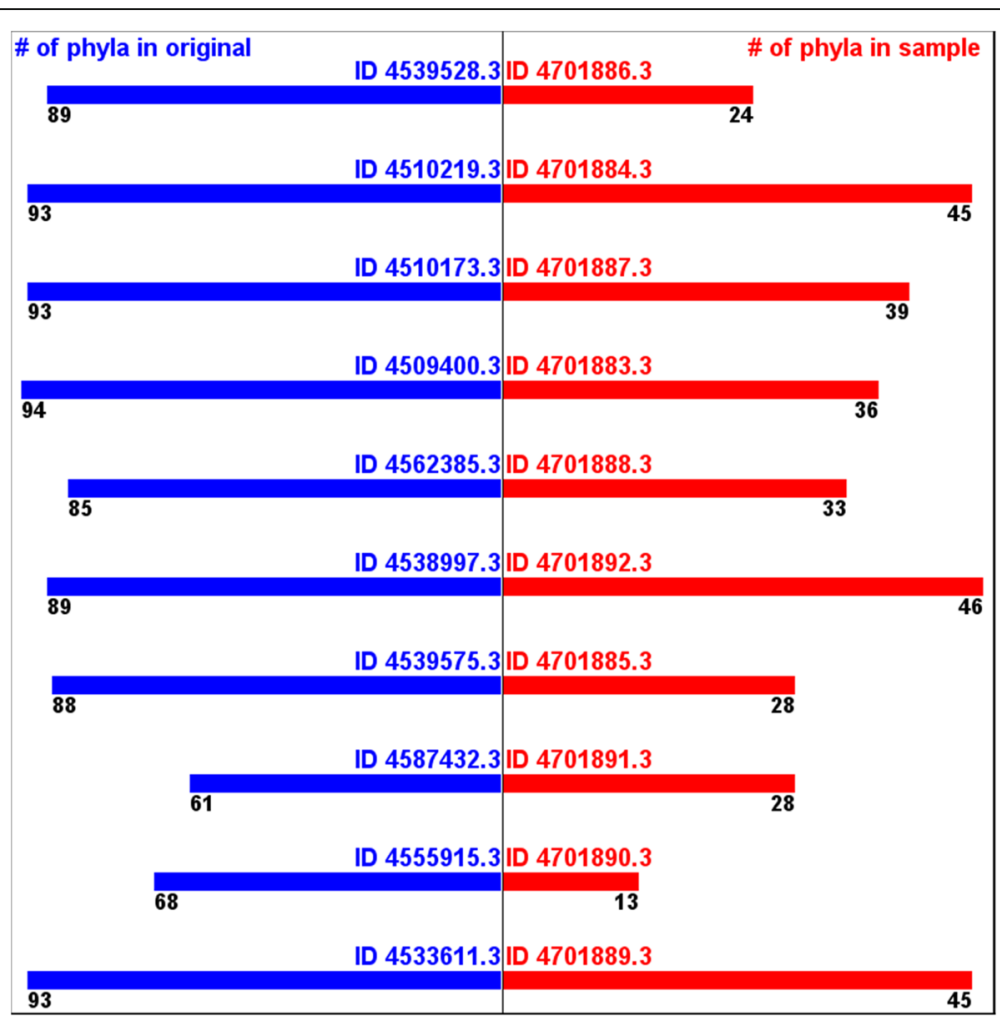

Fig. 4 Numbers of annotated phyla -originals vs. samples

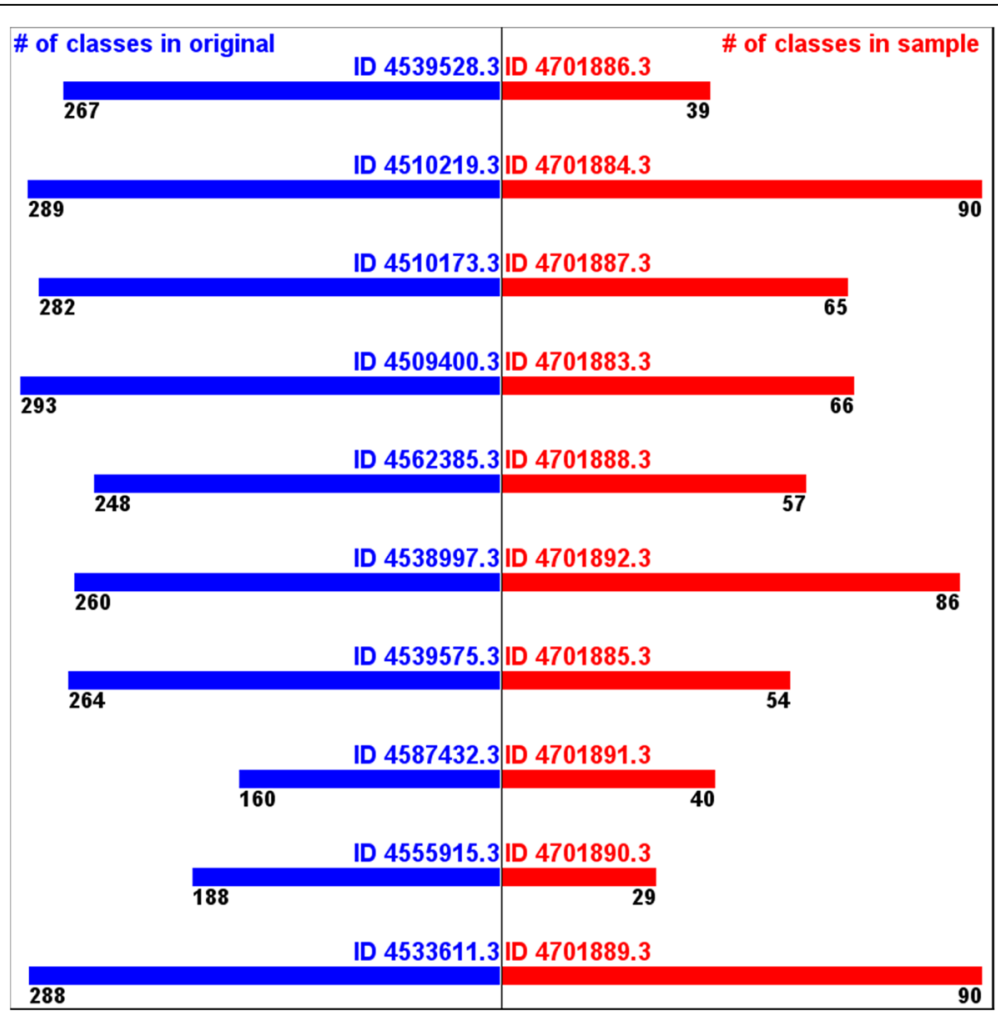

Fig. 5 Numbers of annotated classes -originals vs. samples 


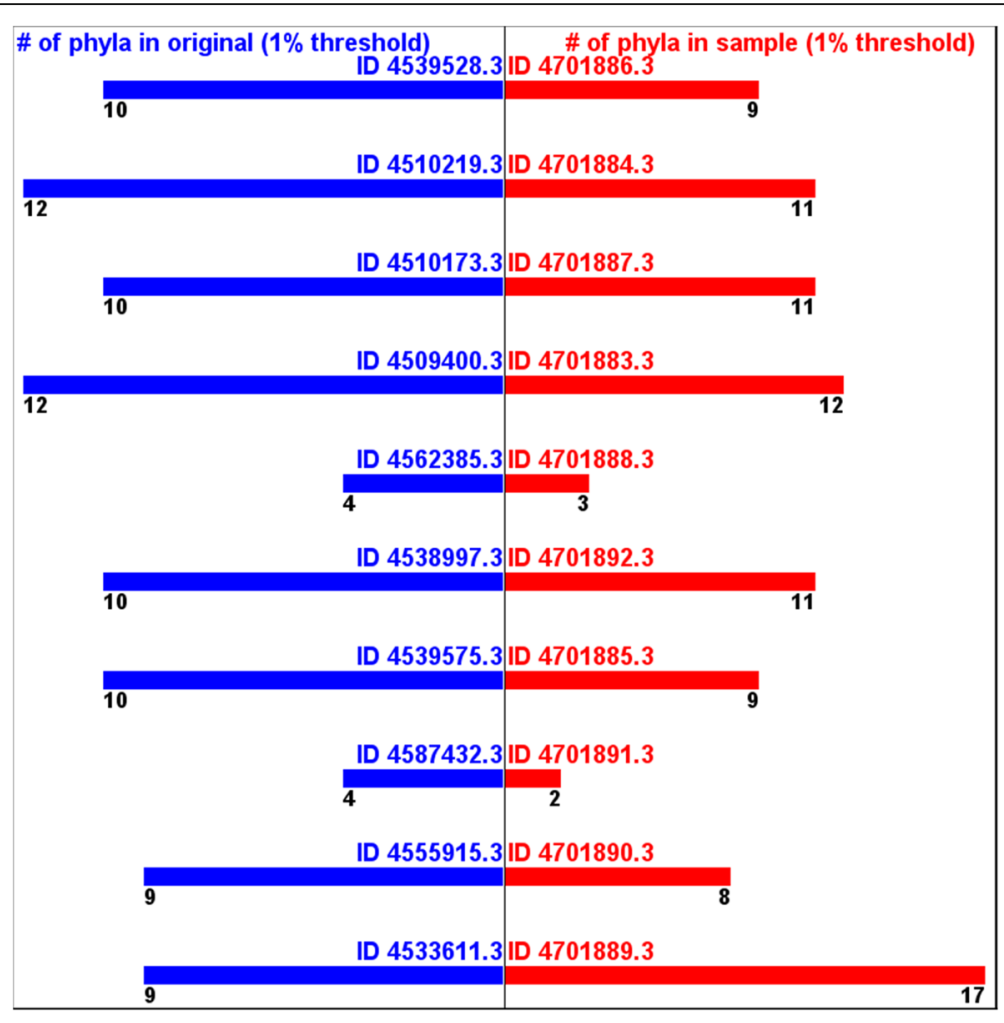

Fig. 6 Numbers of annotated phyla ( $1 \%$ threshold) -originals vs. samples

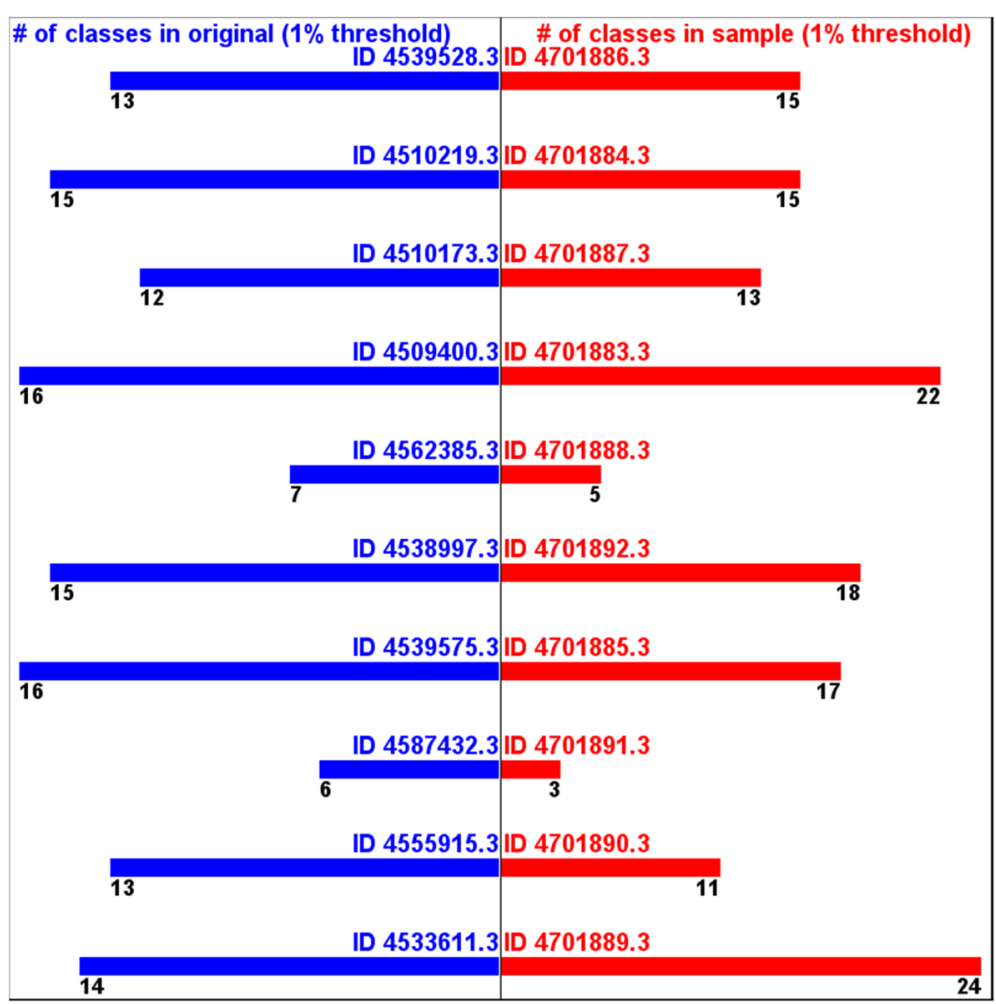

Fig. 7 Numbers of annotated classes (1\% threshold) -originals vs. samples 


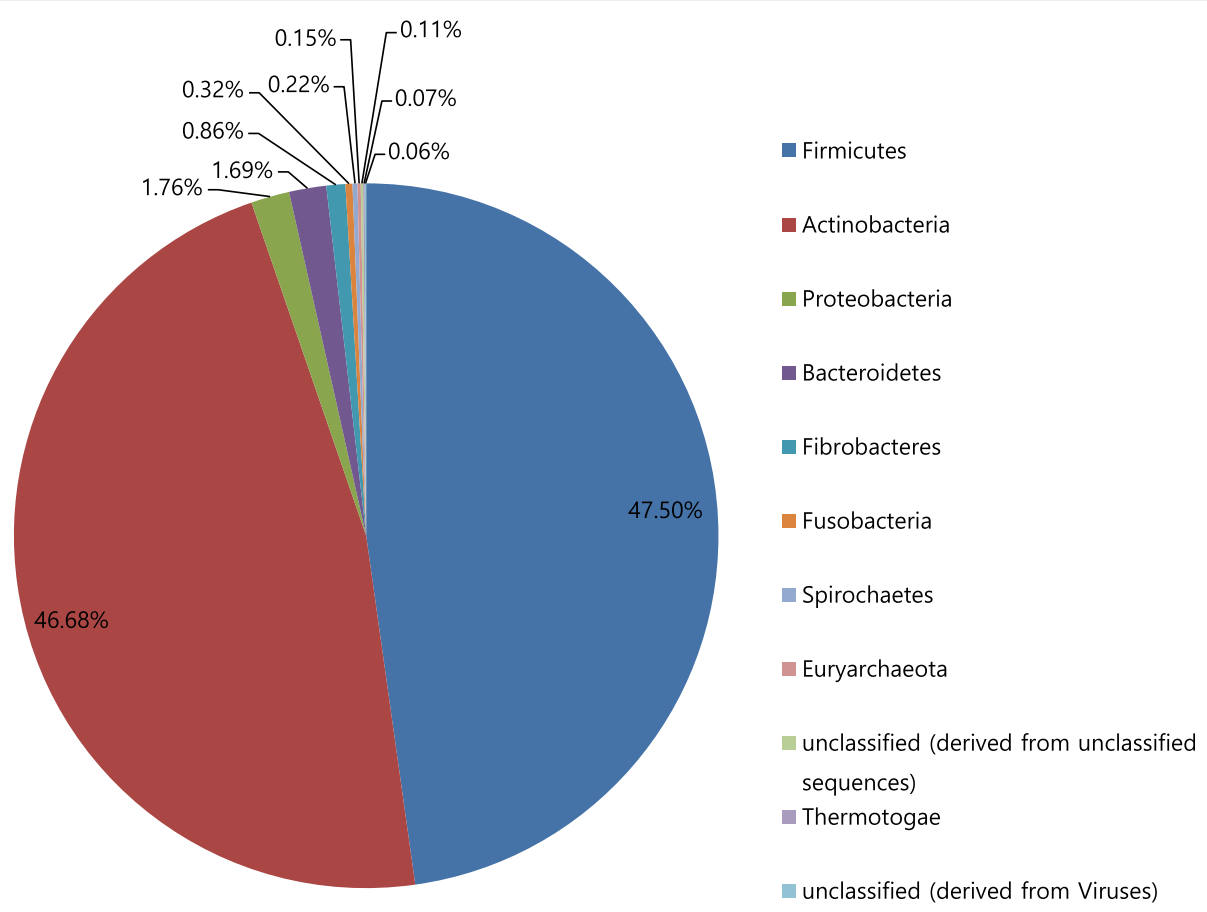

Fig. 8 Phyla annotated from original - MG-RAST ID:4587432.3

of covering the studies of various phyla and classes. More tests for different metagenomics studies covering more divergent environment might be necessary.

In addition, tests of taxonomic annotation not only for phyla and classes but also for genus and species need to be followed in order to know the applicability of this sampling approach better. Another type of sequence analysis rather than taxonomy annotation needs to be tested, too.

Further quantitative studies to suggest statistical criteria of a sample size, as well as studies of how to apply quality filtering to sample sequences, will also make the approach described here more reliable.

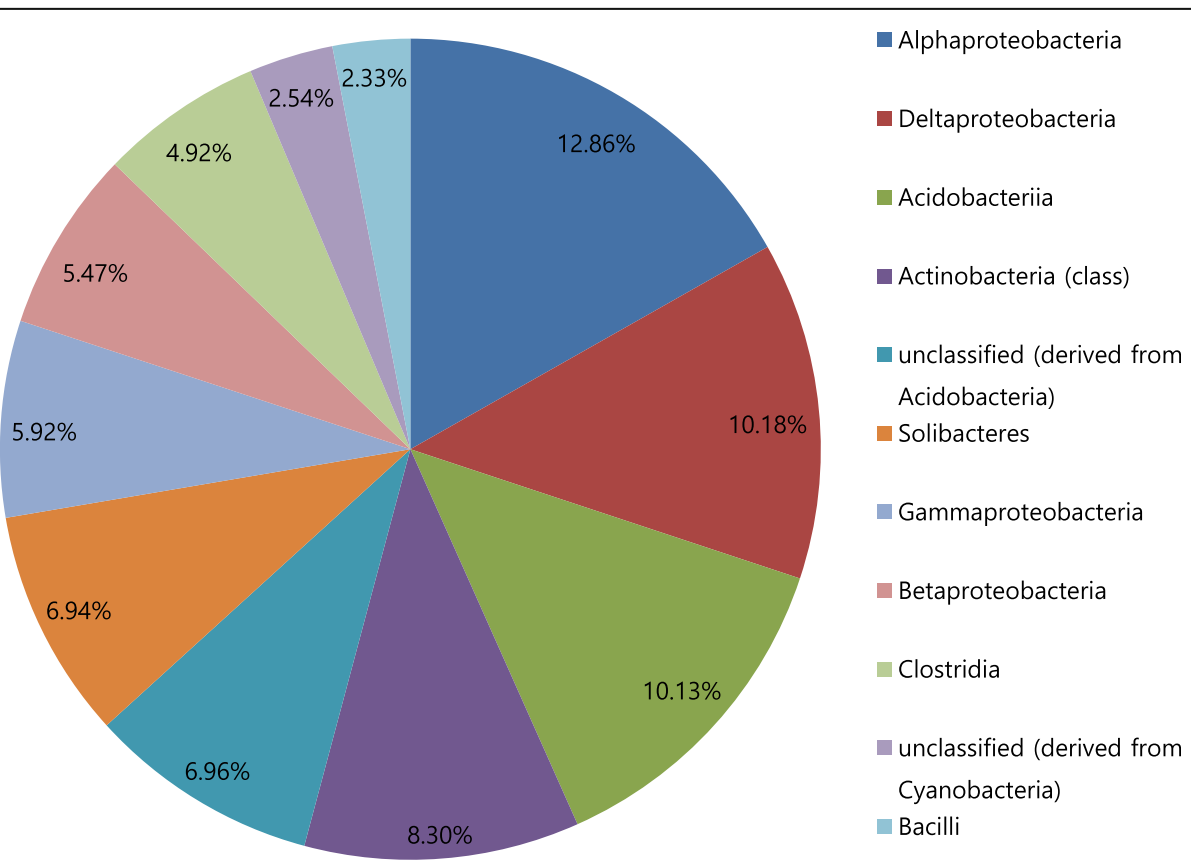

Fig. 9 Classes annotated from original MG-RAST ID:4538997.3 


\section{Conclusions}

In spite of this obvious statistical limit, since analysis from a small size sample of metagenomic sequences only takes short time and uses small computing resources, we can still use this approach to develop a standard or a protocol to preview or pre-check metagenomics data, before performing more accurate analysis with original full sequences.

If there is a case where even a brief information of taxonomic distribution is important, we can use estimation by sample to study a biosample much quickly or to study multiple biosamples as many as possible. For example, we can suggest a strategy of metagenomics research, such as analyzing many biosamples quickly or frequently with small samples of sequences, as the first step of screening, and as the second step, analyzing full original sequences of a few biosamples that showed significant characters at the first step.

If we apply this strategy to assessment of soil pollution with bacteria diversity or to assessment of human health with gut microbiota [18], we can screen out unpolluted locations/low risk cases with this quick sample analysis, and can perform more accurate original full sequence analysis only for suspicious locations/cases. We might perform small size sample studies to monitor bacterial diversities of 100 or 1000 spots, covering a whole state or a nation, on a monthly or even on a weekly basis to discover and track environmental change.

Similarly, we can build a strategy to get taxonomic information of bacteria quickly for forensic studies $[19,20]$ to save time for a criminal investigation. This approach will be also helpful in developing countries where the cost of computing resources is relatively heavy.

\section{Methods}

\section{Mock community}

The mock community with 10 bacteria strains was prepared and their mixed genomes were shotgun sequenced by Hiseq. (Table 4) They are the identical data prepared for a study of Shin S [21].

Then, we selected only small parts from the original full sequences $(1,220,000$ reads), which were named as

Table 4 Strains of the mock community

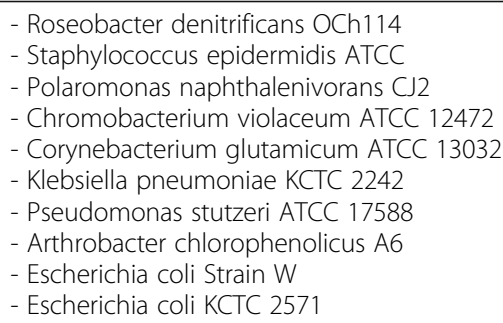

"sample sequence set"s or "sample"s. We generated 176 different samples, in total, that are in 11 different sample sizes. For each sample size, we tried 16 different sampling methods. $(176=11 \times 16)$ The minimum size of the sample was 100 reads $(10,100 \mathrm{~b})$ and the maximum size of the sample was 50,000 reads $(5,050,000 \mathrm{~b})$.

The sampling methods are categorized as 4 selection types, which are:

1. Selecting the reads from the start, after skipping $\mathrm{K}$ number of the reads

2. Selecting the reads from the end, after skipping $K$ number of the reads

3. Selecting the reads from uniformly distributed positions, after skipping $\mathrm{K}$ number of the reads

4. Random selection of the reads

We tried 4 different "K number"s for each type. The random selection was tried for 4 different random seeds. Therefore, the 16 different sampling methods applied to each size of the samples.

To review the samples, we calculated GC content, which is one basic way to know the quality of each sample [22].

To get information about taxonomic annotation, we performed a simple BLAST search for the entire sequences of the mock community with respect to the reference genome databases of the strains [23]. BLAST is a widely used software that can search a query sequence out of a reference genome database. Therefore, if there is a given read of metagenome sequences, a researcher can perform a search to know whether it is found as a hit in a reference genome database or not. In this study, BLAST 2.3.0+ was used, with E-value option of 1e-10. The reference genome databases were downloaded from GeneBank [24].

We performed the BLAST search for every single read of the sequences of the mock community with reference genome database for each of all 10 strains. The number of the hits (denoted as $n i$ ) for genome database of each strain was counted. After all the searches were completed, the sum (denoted as $s$ ) of all the numbers of the hits counted for all 10 strains was calculated. $\left(s=\sum n i\right)$ Then, the ratio $(n i / s)$ of each strain's hit to the sum was also calculated.

To get the information about the taxonomic annotation from the samples, we, again, performed BLAST search for each sample, in the same way as we did for the original full sequences.

The purpose of this ratio calculation is to do simple comparison between the numbers of the hits from the original full sequences and the numbers of the hits from the samples, not getting the actual information about taxonomic abundance. Therefore, the size difference between reference genomes were not considered. 
Table 5 Public MG-RAST projects with 10 most sequences

\begin{tabular}{|c|c|c|c|c|c|}
\hline $\begin{array}{l}\text { Original } \\
\text { MG-RAST ID }\end{array}$ & Project Name & Title of Sequences & Biome & Feature & Material \\
\hline $4,539,528.3$ & GP corn unassembled & iowa-corn-GAll-round1 & terrestrial biome & terrestrial habitat & soil \\
\hline $4,510,219.3$ & $\begin{array}{l}\text { Penang Mangrove } \\
\text { Metagenome }\end{array}$ & BatuMaung_Penang & Mangrove Biome & Wetland & Peat soil \\
\hline $4,510,173.3$ & BP_Sediments & $\begin{array}{l}\text { 2011-1933_120131_SN1035_0095_- } \\
\text { BD04PVACXX_S_4_sequence.fasta }\end{array}$ & marine benthic biome & ocean & $\begin{array}{l}\text { marine } \\
\text { sediment }\end{array}$ \\
\hline $4,509,400.3$ & $\begin{array}{l}\text { Hofmockel Soil Aggregate } \\
\text { COB KBASE }\end{array}$ & $\begin{array}{l}\text { PF41-LM-July2012 / H14_ACTT } \\
\text { GA_L007 }\end{array}$ & Temperate grasslands & terrestrial habitat & agricultural soil \\
\hline $4,562,385.3$ & D.I. Tarballs 0610 & D.I.Tarball 0610 & aquatic biome & $\begin{array}{l}\text { mesoscopic } \\
\text { physical object }\end{array}$ & $\begin{array}{l}\text { organic } \\
\text { material }\end{array}$ \\
\hline $4,538,997.3$ & $\begin{array}{l}\text { Marcell Experimental Forest } \\
\text { carbon cycling }\end{array}$ & MG-T3F-75cm_pair_retain & $\begin{array}{l}\text { Temperate needle-leaf } \\
\text { forests or woodlands }\end{array}$ & forest & soil \\
\hline $4,539,575.3$ & GED prairie unassembled & 1461.5.1405 trimmed & terrestrial biome & terrestrial habitat & soil \\
\hline $4,587,432.3$ & $\begin{array}{l}\text { HMP SRP002423 Bacterial } \\
\text { Fungal Taxonomic Analysis }\end{array}$ & SRS301868_joined & terrestrial biome & $\begin{array}{l}\text { human-associated } \\
\text { habitat }\end{array}$ & feces \\
\hline $4,555,915.3$ & $\begin{array}{l}\text { Beijing Hospital Air } \\
\text { MetaGenome Part1 }\end{array}$ & ICU-Intensive Care Unit & terrestrial biome & air conditioning unit & air \\
\hline $4,533,611.3$ & & M2_retainMode & & & \\
\hline
\end{tabular}

\section{MG-RAST}

To estimate the brief taxonomic information of the original full sequences, denoted as "original" or "original data" hereafter, in actual cases of bacteria metagenomes, we prepared the samples from the existing research data of metagenomics which were publicly available in MG-RAST [15].

Our purpose was to see how this type of the samples can capture the information of the original data in different real world metagenomics studies. MG-RAST has been a tool allows an external researcher to access the results of existing metagenomics studies already performed before.

We downloaded the sequence files of the public MG-RAST projects with 10 most sequences. (Table 5).

Preparing the selected parts of the originals, in other words, the samples, we selected 5000 reads from the start of each original full sequences. (Table 6) This means we used the sampling method of "selection type 1 " and 0 as "K number".

The only reason why we used this sampling method among other selection types/K numbers was that downloading and handling the entire original sequence data files of these real world studies was too heavy task. If we use selection type 1 and $0 \mathrm{~K}$ number, then handling the entire original sequence data files is not necessary. We can just download only the beginning parts of the data files and use them as samples. This reduces sampling cost in terms of data preparation and data handling.

To compare the taxonomic annotation between the originals and the samples, we uploaded the samples to MG-RAST and analyzed them, using MG-RAST, as its available version, that is MG-RAST pipeline 3.6 with its default options. After analysis, MG-RAST gives a list of annotated phyla, classes, and the numbers of how many times they are annotated, as a result.

Table 6 Original full sequences and samples from MG-RAST

\begin{tabular}{llllll}
\hline Original MG-RAST ID & \# Of b.p. in Original & \# Of Reads in Original & Sample MG-RAST ID & \# Of b.p. in Sample & \# Of Reads in Sample \\
\hline $4,539,528.3$ & $37,968,936,507$ & $520,346,510$ & $4,701,886.3$ & 306,684 & 5000 \\
$4,510,219.3$ & $56,396,775,865$ & $419,709,973$ & $4,701,884.3$ & $1,011,153$ & 5000 \\
$4,510,173.3$ & $37,220,314,566$ & $368,517,966$ & $4,701,887.3$ & 505,000 & 5000 \\
$4,509,400.3$ & $28,875,056,044$ & $285,891,644$ & $4,701,883.3$ & 505,000 & 5000 \\
$4,562,385.3$ & $30,079,534,981$ & $269,568,253$ & $4,701,888.3$ & 749,082 & 5000 \\
$4,538,997.3$ & $36,830,201,101$ & $242,692,675$ & $4,701,892.3$ & $1,179,840$ & 5000 \\
$4,539,575.3$ & $19,954,890,565$ & $233,720,367$ & $4,701,885.3$ & 434,006 & 5000 \\
$4,587,432.3$ & $24,845,881,691$ & $220,848,213$ & $4,701,891.3$ & 671,320 & 5000 \\
$4,555,915.3$ & $17,546,603,952$ & $173,728,752$ & $4,701,890.3$ & 505,000 & 513,458 \\
$4,533,611.3$ & $17,299,825,549$ & $172,590,841$ & $4,701,889.3$ & 5000 & 5000 \\
\hline
\end{tabular}


Since, the original data were from the publicly accessible project, we can also access the analysis results of them, already performed before. Therefore, we can get the lists of annotated phyla, classes and the numbers of how many times they were annotated for the original data, too.

Then, the most annotated phyla from the originals and the most annotated phyla from the samples were compared, as well as the most annotated classes from the originals and the ones from the samples.

To compare taxonomic diversities, the number of the annotated phyla and classes was counted for each of the originals and for each of the samples. Considering over-annotation and/or mis-annotation of MG-RAST, a threshold to ignore phyla and classes with less than $1 \%$ hits of the total hits was later applied, in accordance with the study of Peabody et al. [3].

\section{Additional file}

Additional file 1: Table S1. The smallest values among the ratios calculated from 16 samples of each sample size ("Ratio from the original" is calculated from the original full sequences, which is same as the value of Table 1). Table S2. The largest values among the ratios calculated from 16 samples of each sample size ("Ratio from the original" is calculated from the original full sequences, which is same as the value of Table 1). Table S3. Standard deviation from the ratios calculated from 16 samples of each sample size. (DOCX $25 \mathrm{~kb}$ )

\section{Acknowledgements}

The sequence data of the mock community were prepared by Dr. Shin S, Environmental Biotechnology Lab, Department of Civil and Environmental Engineering, Yonsei University. This work was supported by Korea Ministry of Environment as "Global Top Project (2016002130005)".

\section{Funding}

No funding was received.

\section{Availability of data and materials}

The datasets used in MG-RAST service during the current study are available in MG-RAST repository, https://www.mg-rast.org.

Other datasets used and/or analysed during the current study are available from the corresponding author on reasonable request.

\section{Authors' contributions}

JK designed the test, performed the calculation, and wrote the manuscript JP reviewed the results, suggested the interpretation, and suggested the forms of the tables and the figures. Both authors read and approved the final manuscript.

\section{Ethics approval and consent to participate}

Not applicable

\section{Consent for publication}

Not applicable

\section{Competing interests}

The authors declare that they have no competing interests.

\section{Publisher's Note}

Springer Nature remains neutral with regard to jurisdictional claims in published maps and institutional affiliations.

\section{Author details}

Graduate Program in Technology Policy, Yonsei University, 50 Yonsei Ro, Seodaemun Gu, Seoul 038722, South Korea. ${ }^{2}$ School of Civil and Environmental Engineering, Yonsei University, 50 Yonsei Ro, Seodaemun Gu, Seoul 038722, South Korea.

Received: 16 April 2018 Accepted: 10 October 2018

Published online: 03 November 2018

\section{References}

1. Vincent AT, Derome N, Boyle B, Culley Al, Charette SJ. Next-generation sequencing (NGS) in the microbiological world: how to make the most of your money. J Microbiol Methods. 2016. https://doi.org/10.1016/j.mimet. 2016.02.016

2. Simon C, Daniel R. Metagenomic analyses: past and future trends. Appl Environ Microbiol. 2011. https://doi.org/10.1128/AEM.02345-10.

3. Peabody MA, Van Rossum T, Lo R, Brinkman FS. Evaluation of shotgun metagenomics sequence classification methods using in silico and in vitro simulated communities. BMC Bioinformatics. 2015. https://doi.org/10.1186/ s12859-015-0788-5.

4. Segata N, Waldron L, Ballarini A, Narasimhan V, Jousson O, Huttenhower C. Metagenomic microbial community profiling using unique clade-specific marker genes. Nat Methods. 2012. https://doi.org/10.1038/nmeth.2066.

5. Wood D, Salzberg SL. Kraken: ultrafast metagenomic sequence classification using exact alignments. Genome Biol. 2014. https://doi.org/10.1186/gb2014-15-3-r46.

6. Kim D, Song L, Breitwieser FP, Salzberg SL. Centrifuge: rapid and sensitive classification of metagenomic sequences. Genome Res. 2016. https://doi. org/10.1101/gr.210641.116

7. Ni J, Yan Q, Yu Y. How much metagenomic sequencing is enough to achieve a given goal? Sci Rep. 2013. https://doi.org/10.1038/srep01968.

8. Caporaso JG, Lauber CL, Walters WA, Berg-Lyons D, Lozupone CA, Turnbaugh PJ, Fierer N, Knight R. Global patterns of $16 \mathrm{~S}$ rRNA diversity at a depth of millions of sequences per sample. Proc Natl Acad Sci U S A. 2011. https://doi.org/10.1073/pnas.1000080107.

9. Poretsky R, LM R-R, Luo C, Tsementzi D, Konstantinidis KT. Strengths and limitations of $16 \mathrm{~S}$ rRNA gene amplicon sequencing in revealing temporal microbial community dynamics. PLoS One. 2014. https://doi.org/10.1371/ journal.pone.0093827.

10. Straub SCK, Parks M, Weitemier K, Fishbein M, Cronn RC, Liston A. Navigating the tip of the genomic iceberg: next-generation sequencing for plant systematics. Am J Bot. 2012. https://doi.org/10.3732/ajb.1100335.

11. Linard B, Crampton-Platt A, Gillett CPDT, Timmermans MJTN, Vogler AP. Metagenome skimming of insect specimen pools: potential for comparative genomics. Genome Biol Evol. 2015. https://doi.org/10.1093/gbe/evv086.

12. Nayfach S, Bradley PH, Wyman SK, Laurent TJ, Williams A, Eisen JA, Pollard KS, Sharpton TJ. Automated and accurate estimation of gene family abundance from shotgun metagenomes. PLoS Comput Biol. 2015. https:// doi.org/10.1371/journal.pcbi.1004573.

13. Kunin V, Copeland A, Lapidus A, Mavromatis K, Hugenholtz P. A bioinformatician's guide to metagenomics. Microbiol Mol Biol Rev. 2008. https://doi.org/10.1128/MMBR.00009-08.

14. Altschul SF, Gish W, Miller W, Myers EW, Lipman DJ. Basic local alignment search tool. J Mol Biol. 1990. https://doi.org/10.1016/S0022-2836(05)80360-2.

15. Meyer F, Paarmann D, D'Souza M, Olson R, Glass EM, Kubal M, et al. The metagenomics RAST server? A public resource for the automatic phylogenetic and functional analysis of metagenomes. BMC Bioinformatics. 2008. https://doi.org/10.1186/1471-2105-9-386

16. Utts J, Heckard R. Cengage Learning. In: Statistical ideas and methods; 2005. Chapter 10. p. 6

17. The New York Times . Latest election polls. The New York Times. 2016. https://www.nytimes.com/interactive/2016/us/elections/polls.html?mcubz=3

18. Sekirov I, Russell SL, Antunes LC, Finlay BB. Gut microbiota in health and disease. Physiol Rev. 2010. https://doi.org/10.1152/physrev.00045.2009.

19. Khodakova AS, Smith RJ, Burgoyne L, Abarno D, Linacre A. Random whole metagenomic sequencing for forensic discrimination of soils. PLoS One. 2014. https://doi.org/10.1371/journal.pone.0104996.

20. Tridico SR, Murray DC, Addison J, Kirkbride KP, Bunce M. Metagenomic analyses of bacteria on human hairs: a qualitative assessment for applications in forensic science. Investig Genet. 2014. https://doi.org/10. 1186/s13323-014-0016-5. 
21. Shin S. Accuracy improvement of next-generation sequencing data through characterization and correction of sequence-specific errors: Graduate school: Yonsei University; 2015

22. Lightfield J, Fram NR, Ely B. Across bacterial Phyla, distantly-related genomes with similar genomic GC content have similar patterns of amino acid usage. PLoS One. 2011. https://doi.org/10.1371/journal.pone.0017677.

23. Sharpton TJ. An introduction to the analysis of shotgun metagenomic data. Front Plant Sci. 2014. https://doi.org/10.3389/fpls.2014.00209.

24. Benson DA, Cavanaugh M, Clark K, Karsch-Mizrachi I, Lipman DJ, Ostell J, GenBank SEW. Nucleic Acids Res. 2013. https://doi.org/10.1093/nar/gks1195.

Ready to submit your research? Choose BMC and benefit from:

- fast, convenient online submission

- thorough peer review by experienced researchers in your field

- rapid publication on acceptance

- support for research data, including large and complex data types

- gold Open Access which fosters wider collaboration and increased citations

- maximum visibility for your research: over $100 \mathrm{M}$ website views per year

At $\mathrm{BMC}$, research is always in progress.

Learn more biomedcentral.com/submissions 